\title{
Ionized Gas Thermoelectric Generator: Theoretical Evaluation and Efficiency Estimation
}

Mohammad Bqoor ${ }^{*}$, Mohammad Hamdan ${ }^{2}$, Isam Janajreh ${ }^{3}$

${ }^{1}$ The University of Jordan, ${ }^{2}$ The University of Jordan, ${ }^{3}$ Khalifa University of Science and Technology

*Correspondence Author, mohd.bqoor@gmail.com

\begin{abstract}
:
The novel Ionized Gas Thermoelectric Generator (IG-TEG) system that has been studied theoretically showing capabilities to continually extracting energy from the thermal energy of the ambient air, at low temperatures within the standard room temperature and below. This system does not need a temperature gradient in order to work, unlike the other TEGs that use Seebeck effect, and therefore this new system can be utilized for cooling purposes, by extracting energy instead of wasting energy in compressing the gas for cooling. This novel system was designed based on Static Ratchet Potential (SRP), which is known as a spatially asymmetric electric potential produced by an array of positive and negative electrodes. The ratchet potential produces electrical current from random Brownian Motion of charged particles that are driven by thermal energy. Ratchet potential was studied and investigated by several researches in the fields of sensing and energy harvesting. The main ratchet potential system parameter is the particles transportation, this parameter was studied under the condition of flashing ratchet potentials, and was analyzed based on several methods. In this study, a different approach is pursued to estimate particles transportation, by evaluating the charged particles distribution, and applying the other conditions of the SRP.
\end{abstract}

\section{Keywords:}

Thermoelectric Generator; Ratchet Potential; Brownian Ratchet; Electrostatic; Particles Distribution; Energy Harvesting. 


\section{Introduction}

The Ratchet Potential (RP) or Brownian Ratchet has been studied and investigated in several researches, its intrinsic core design characterized by Feynman Ratchets ${ }^{[1]}$, that is proposed to extract energy from random Brownian motion of gas particles ${ }^{[2-4]}$. The Ratchet Potential working principle is the directional transport of classical or quantum particles in systems that are dominated by random diffusion ${ }^{[5]}$. This directional transport has been gaining much research interest in the application of ratchet in sensing ${ }^{[6]}$, energy harvesting ${ }^{[7,8]}$ and separation ${ }^{[9]}$. Ratchet Potential was also implemented in many microbiology studies including micro-machine as in the DNA transport and separation ${ }^{[9]}$, the translocation of proteins across membranes ${ }^{[10]}$ and bimolecular machine ${ }^{[9]}$, nanotechnology and nanofluid preconcentration and separation ${ }^{[12]}$, particles motion against ${\text { constant } \text { bias }^{[13]} \text {, Brownian Motors }}^{[14,15]}$, additional to infusion and flow control of nanoscale objects in fluids ${ }^{[16]}$.

Ratchet potential research has been focusing on the Brownian Ratchet implementing flashing potentials and the driving forces ${ }^{[17-19]}$. The particles transport in ratchet potential was considered thoroughly in these researches. Many statistical approaches were implemented to evaluate and generate transportation equations. Amongst those are Fokker-Planck equation to evaluate the time evolution of the probability density function ${ }^{[20]}$, Fick-Jakobs kinetic equation, and the Gaussian white noise ${ }^{[21]}$, as well as the steady-state Smoluchowski equations ${ }^{[22]}$. The flashing ratchet potential and the cyclic distribution of free charged particles, and to the best of authors' knowledge, was not treated thoroughly in the literature, on the contrary of the transportation of particles.

Thermoelectric Generators (TEG) is a device converting thermal energy into electric energy, the common technique used in this device is called "Seebeck effect" ${ }^{[*]}$, which is the production of electric current induced by a temperature gradient between two electrical conductors or semiconductors. A new design of Ionized Gas Thermoelectric Generator (IG- TEG) based on RP technique was theoretically introduced in a previous study ${ }^{[23]}$, the calculations showed the success of this system in converting "wasted" thermal energy into kinetic energy. The previous study imposed several conditions and parameters, and showed several improvements opportunities to be a new competing technology.

This work intended to thoroughly verify the operation methodology and its relevant parameters, and assess the calculated efficiency of the IG-TEG, starting by evaluating the charged Particles Distribution Function (PDF) inside the Static Ratchet Potential (SRP) that characterized by the fixed electric charge imposed on its electrodes, while keeping its geometry similar to that of the flashing ratchet. Then exploiting this function in the IG-TEG functionality examination, such examination will extend to particles transportation and fluid dynamics calculations inside the SRP. This work is aiming to conduct a deep analysis of the novel IG-TEG and assess the validity of the $2^{\text {nd }}$ Law of Thermodynamics ${ }^{[24,25]}$.

\section{Design, Methodology and System Setup}

The flow dynamics and its characteristics inside the SRP require an accurate estimation of PDF. This estimation can be done by using Gauss's flux theorem ${ }^{[26]}$. Considering a system of $N$ free charged particles at a temperature $T=0 \mathrm{k}$ distributed inside a given volume, the particles are 
expected to be arranged in stationary locations producing a zero net electric force ${ }^{[27]}$. This reference state is referred to as the equipotential state $(\nabla V=0)$. The charged particles will be assumed protons (ionized hydrogen $\mathrm{H}^{+}$) and their distribution can be represented as per Fig. 1 and based on the equipotential locations. The linear distribution of positive particles can be also extrapolated to volumetric distribution by keeping their uniformity in $y-z$ plane and vary their distribution along the $x$-axis.

All positive particles will be laid in equipotential locations as depicted in Fig.1. The equipotential value $V_{E q u}\left(x_{j}\right)$ for a number of positive particles $N$ in one-dimensional ( $x$-axis) distribution can be written based on the scalar summation of electric potentials at the $x_{j}$ location $^{[27]}$, which is written as:

$$
V_{E q u}\left(x_{j}\right)=\frac{1}{4 \pi \epsilon_{o}} \sum_{\substack{i=1 \\ i \neq j}}^{N} \frac{q}{\left|x_{j}-x_{i}\right|}+V\left(x_{j}\right)
$$

Where $V\left(x_{j}\right)$ is the electrodes' potential of the SRP as shown per Fig.1, and is given as:

$$
V(x)_{\text {Electrodes }}=\frac{1}{4 \pi \epsilon_{o}}\left(\sum_{n=-M}^{M}\left(\frac{-Q}{\sqrt{(x-n D)^{2}+a^{2}}}\right)+\sum_{n=-M}^{M}\left(\frac{+\alpha Q}{\sqrt{(x-n D+d)^{2}+a^{2}}}\right)\right)
$$

Where $M$ is the number of a few SRP's electrodes that can be considered in a central axis, $\alpha$ is the ratio of charges that are mounted in positive electrodes to those in negative electrodes. In reference to Fig. 1, the number of positive particles (red, green and yellow circles) will determine the equipotential value $\left(0, V_{1}, V_{2}\right)$, and these values determine the borders of the $\operatorname{PDF}\left(x_{1}, x_{2}, \ldots, x_{6}\right)$.

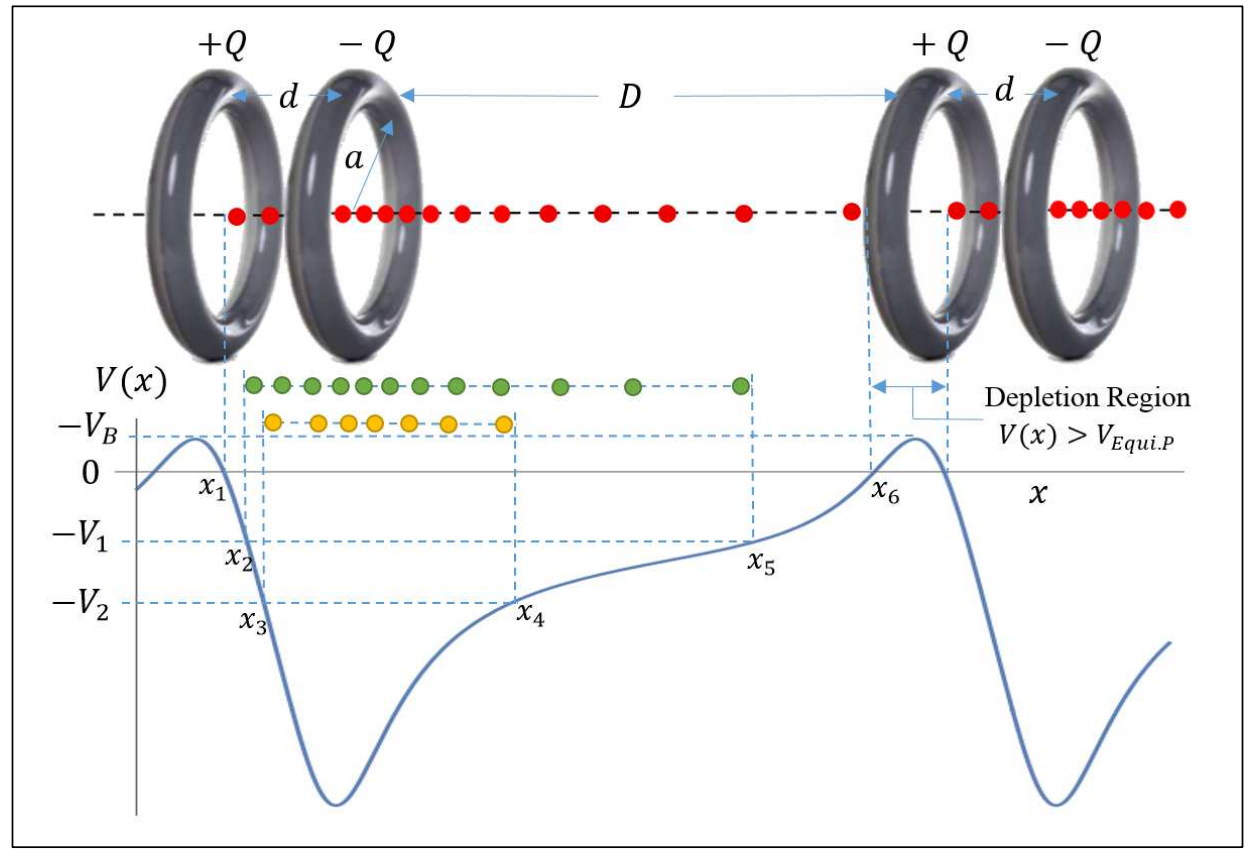

Fig.1: Illustration of positive particles distribution and the ratchet parameters 
Table.1 provides realistic values to the numerous parameters appear in Equation (2). The parameters was chosen based on rational values of the methods of microelectrodes manufacturing capabilities $^{[28]}$ and material electrical properties, mainly the voltage break-down of dielectric coating materials ${ }^{[29]}$.

Table.1: Summary of the SRP parameters and their values

\begin{tabular}{|l|c|l|c|}
\hline \multicolumn{1}{|c|}{ Parameter } & Value (Unit) & \multicolumn{1}{c|}{ Parameter } & Value (Unit) \\
\hline $\begin{array}{l}\text { Number of electrodes pairs } \\
(M)\end{array}$ & 11 & $\begin{array}{l}\text { Distance between adjacent } \\
\text { similar electrodes }(D)\end{array}$ & $5(\mathrm{~mm})$ \\
\hline $\begin{array}{l}\text { Radius of electrode ring } \\
(a)\end{array}$ & $0.5(\mathrm{~mm})$ & $\begin{array}{l}\text { Negative charge mounted in } \\
\text { negative electrodes }(-Q)\end{array}$ & $1 \times 10^{-10}(C)$ \\
\hline $\begin{array}{l}\text { Distance between adjacent } \\
\text { opposite electrodes }(d)\end{array}$ & $1(\mathrm{~mm})$ & $\begin{array}{l}\text { Positive to negative charge } \\
\text { ratio }(\alpha)\end{array}$ & $60 \%$ (percentile) \\
\hline
\end{tabular}

The electric potential produced by the linear charge density of positive particles is depicted in Fig2. To have the positive particles at equipotential locations $\left(V_{e q}=0\right)$, then the electric potential produced by the distribution of positive particles $\left(V(x)_{\text {Positive Particels }}\right)$ should be a mirror symmetry of the electrodes potential $\left(V(x)_{\text {Electrodes }}\right)$ over the $x$-axis, i.e. mathematically:

$$
\begin{gathered}
V_{e q}=V(x)_{\text {Positive Particels }}+V(x)_{\text {Electrodes }}=0 \text { or } V(x)_{\text {Positive Particels }}=-V(x) \\
x \in\left\{x_{1}, x_{6}\right\}
\end{gathered}
$$

Accordingly, and from Equation (2):

$$
\begin{gathered}
V(x)_{\text {Positive Particels }}=\frac{1}{4 \pi \epsilon_{o}}\left(\sum_{n=-M}^{M}\left(\frac{-Q}{\sqrt{(x-n D)^{2}+a^{2}}}\right)+\sum_{n=-M}^{M}\left(\frac{+\alpha Q}{\sqrt{(x-n D+d)^{2}+a^{2}}}\right)\right) \\
x \in\left\{x_{1}, x_{6}\right\}
\end{gathered}
$$

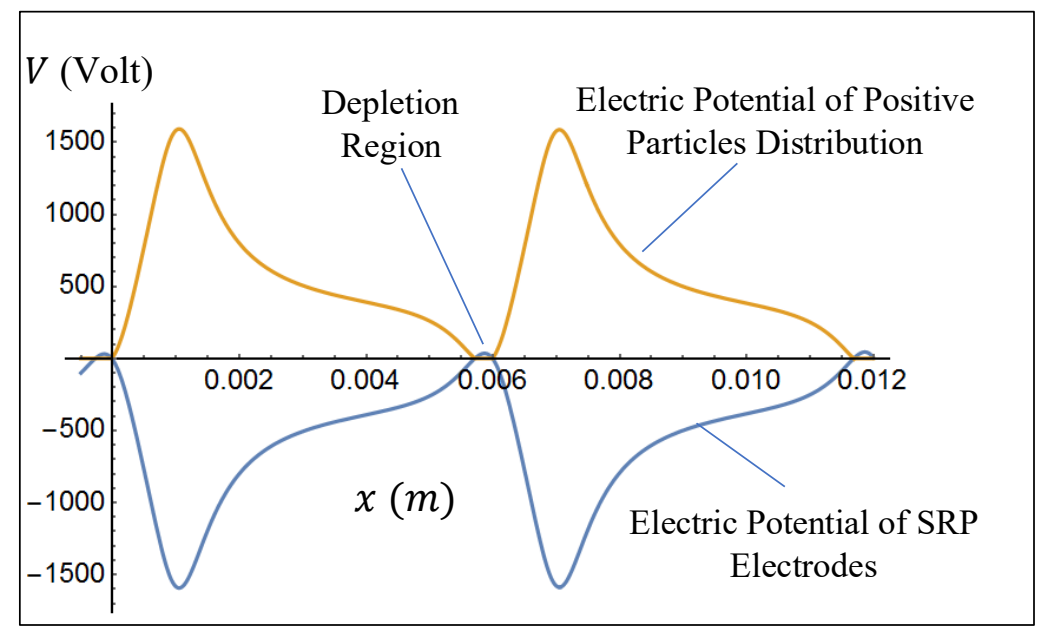

Fig.2: Electric potential of the SRP electrodes and positive particles distribution. 
As seen in Fig.2, the summation of the Electric Potential of PDF and SRP electrodes is nil $(V=0)$ except at the depletion regions. Therefore, it is very obvious that the electric potential shape and the magnitude of SRP electrodes will formulate that of the PDF.

\section{Results and Discussion}

Energy harvesting as the intended application of SRP relies on the electrical and geometrical factors of the system ${ }^{[23]}$. These factors are determining both the number of these particles and their distribution (PDF). Since the thermal energy of the ionized gas positive particles can be extracted by the directional transport of these particles, the number of positive particles and PDF are significantly affecting the energy harvesting efficiency of the SRP.

To evaluate the PDF, the total number of positive particles needs to be determined. This number depends on the equipotential value of the positive particles. For sake of simplicity, the positive particles will be assumed to be located at $V=0$ equipotential locations. Hence, and in reference to Fig.2, the positive charge will be distributed in the allotted semi-cylindrical volume $\pi a^{2} x_{6}-$ $x_{1}$ ), where the boundaries of the PDF is bounded at $x_{1}=-0.0138 \mathrm{~mm}$ to $x_{6}=5.714 \mathrm{~mm}$, and maintaining the equipotential condition $\left(V_{E q u}=0\right)$ in every positive particle location within the semi-cylindrical space.

\subsection{Number of Free Positive Particles}

To evaluate the total number of positive particles $\left(N_{P}\right)$, the cylindrical surface shown in Fig.3 is chosen as Gaussian surface and this formulated as ${ }^{[27]}$ :

$$
\frac{Q_{e n c}}{\epsilon_{o}}=\oint E(x) \cdot d A=\oint-\nabla V \cdot d A
$$

The static condition of positive particles in the equipotential locations $(V=0)$ on the Gaussian surface implies uniform localized voltage $(\nabla V=0)$ in all directions. Then evaluating Equation (5) on the considered cylindrical surface, leads to:

$$
\frac{Q_{\text {enc }}}{\epsilon_{o}}=0
$$

Hence, the total charge of positive particles will be simply the difference in the charge between positive and negative electrode, and this is calculated leading to:

$$
Q_{\text {Positive Particels }}=(1-\alpha) Q=0.4 \times 10^{-10} \mathrm{C}
$$

The total number of positive particles will be evaluated based on the unit charged particle $\left(1.6 \times 10^{-19} \mathrm{C}\right)$ as:

$$
N_{P}=\frac{0.4 \times 10^{-10}}{1.6 \times 10^{-19}}=2.5 \times 10^{8} \text { Particles }
$$




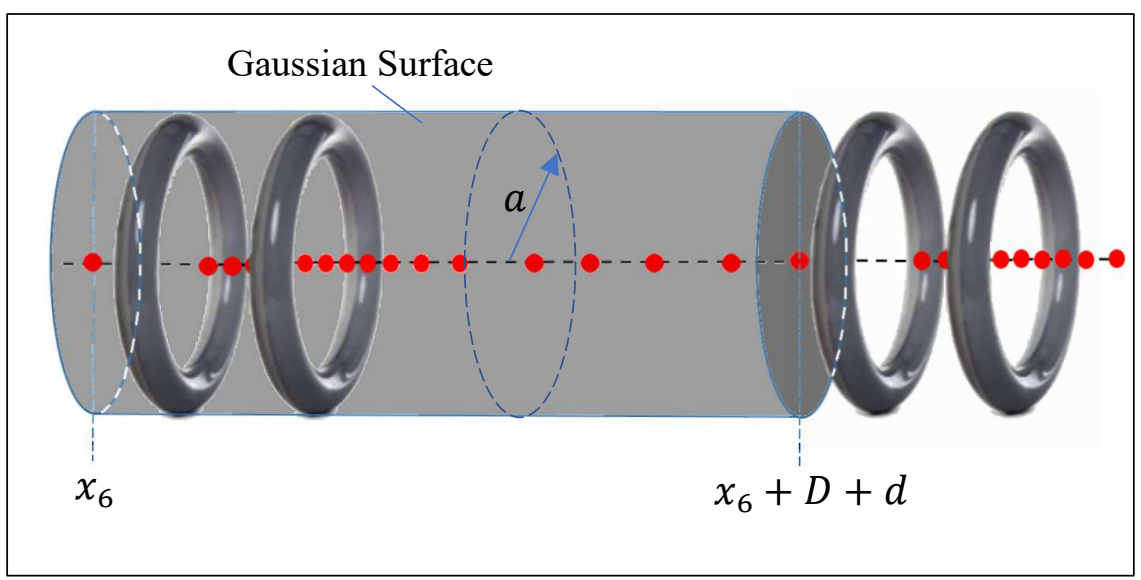

Fig.3: The cylindrical Gaussian Surface with dimensions and geometrical boundaries: cylinder radius is $(a)$, and their bases are located at $\left(x=x_{6}\right)$ and $\left(x=x_{6}+D+d\right)$.

\subsection{Verification of the Total Number of Free Positive Particles}

This is achieved by considering the average distance between positive particles and assuming a simple (Primitive) cubic lattice ${ }^{[30]}$ (SCL) arrangement of positive particles. Referring to Fig.2, the "linear-average" of the electric potential $\left(\bar{V}_{\text {Positive Particels }}\right)$ can be evaluated as:

$$
\begin{gathered}
\bar{V}_{\text {Positive Particels }}= \\
\frac{1}{x_{6}-x_{1}} \frac{1}{4 \pi \epsilon_{o}} \int_{x_{1}}^{x_{6}} \sum_{n=-M}^{M}\left(\frac{-Q}{\sqrt{(x-n D)^{2}+a^{2}}}\right)+\sum_{n=-M}^{M}\left(\frac{+\alpha Q}{\sqrt{(x-n D+d)^{2}+a^{2}}}\right) d x
\end{gathered}
$$

This gives an average electric potential in the range 612 volts. The average spacing or the SCL length $(\bar{l})$ between positive particles can be geometrically calculated as:

$$
\bar{l} \cong \sqrt[3]{\frac{\pi a^{2}(D+d)}{N_{P}}}=\sqrt[3]{\frac{\pi(0.0005)^{2}(0.005+0.001)}{2.5 \times 10^{8}}} \cong 2.66 \times 10^{-6} \mathrm{~m}
$$

Then determining the electric potential at the central positive particle in SCL arrangement by having the cubic root of $N_{p}$ such that:

$$
\frac{\sqrt[3]{N_{p}}}{2} \cong 315
$$

The positive particles in each side of Cartesian coordinated $(x, y$ and $z$ ) will form the eight Cartesian blocks. Hence, the average positive particle potential is evaluated from the triple summation of the Madelung Constant ${ }^{[31]}$ of similar charge sign particles, as: 


$$
\bar{V}_{\text {Positive Particels }}=\frac{8}{4 \pi \epsilon_{o}} \sum_{m=0}^{315} \sum_{n=0}^{315} \sum_{p=1}^{315} \frac{1.6 \times 10^{-19}}{\sqrt{(m l)^{2}+(n l)^{2}+(p l)^{2}}} \cong 514 \text { Volts }
$$

The order of this obtained value is in general agreement with the result achieved from Equation (9). Specifically, the (16\%) difference in resulting potential evaluated in Equations (9) and (11) is due to the "linear-averaging" of the "non-linear" curve of the positive particles distribution electric potential which was shown in Fig. 2.

\subsection{Evaluation of the Positive Particles Distribution Function}

The relation between electric potential produced by number $(N)$ of positive particles distributed in fixed SCL arrangement is almost linear for $(N \gg 1)$ in Equation (11). This is due to the fact that as $N$ is increasing the more linear the curve becomes as shown in fig. 4.

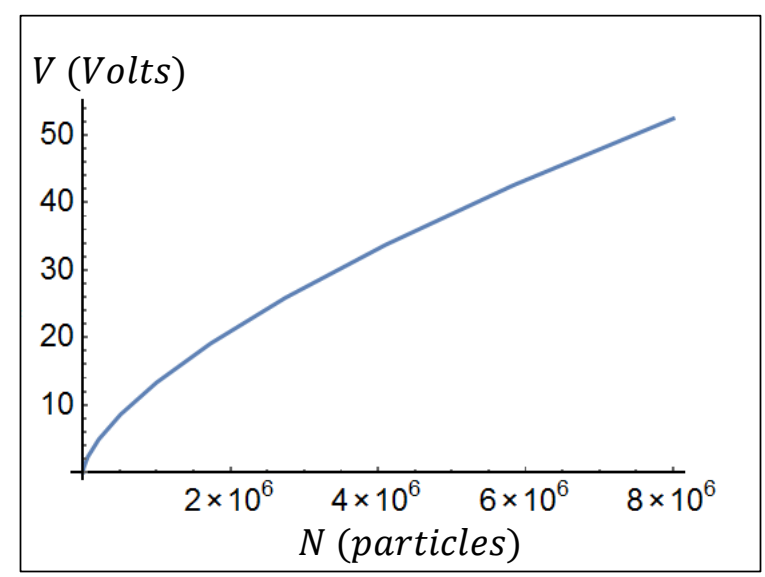

Fig.4: Electric potential produced by positive particles in fixed SCL arrangement, with equal spacing $\left(2.66 \times 10^{-6} \mathrm{~m}\right)$ as evaluated in Equation (10).

The linear relation for $(N \gg 1)$ in Fig.4 is further explained such that the electric potential $\bar{V}_{\text {Positive Particels }}$ dependence on the distance between central particles and other particles $\left(r=\sqrt{(m l)^{2}+(n l)^{2}+(p l)^{2}}\right)$ which is in the order of $\left(r^{-1}\right)$. The number of positive particles $N(r)$ located at $r$ distance will depend on the surface of the sphere of radius $r$ where the positive particles are proportional to its surface $\left(N(r) \propto 4 \pi r^{2}\right)$. The additional positive particles will be located at the same distance from the central particle, i.e. at the surface of a sphere as shown in Fig.5. Thus, the relation between the produced electric potential $\bar{V}_{\text {Positive Particels }}$ and the number of positive particles $N$ will be as $\left(r^{-1} \cdot r^{2}=r\right)$, i.e. the linear relation shown in Fig.4. 


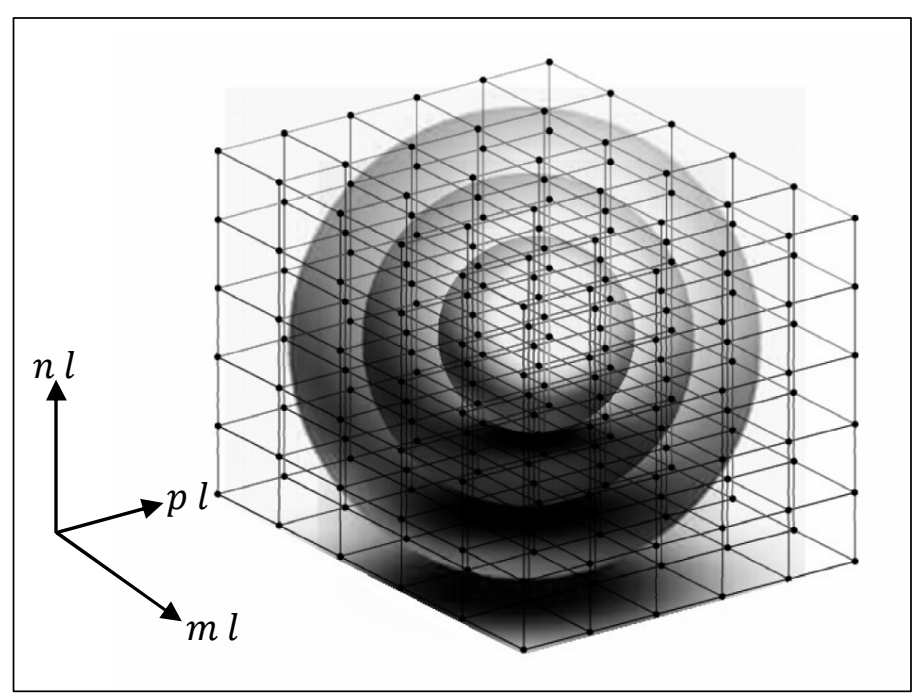

Fig.5: Positive particles in fixed SCL, and the number of positive particles that will be laid at the same distance from the central particle.

Because of the linear relation between the produced electric potential and the number of positive particles, the PDF can be evaluated from Fig.2 by changing the scale of $y$-aixs of the electric potential of the PDF (the yellow curve), this can be done by introducing a normalization factor $(C)$ such that:

$$
N_{P}=\int_{x_{1}}^{x_{6}} \sigma(x) d x=C \int_{x_{1}}^{x_{6}} V(x)_{\text {Positive Particels }} d x
$$

Substituting $V(x)_{\text {Positive Particels }}$ of Equation (4) will lead to determining the normalization constant as $C=7.12 \times 10^{7}$. Therefore, the PDF is formulated according to Equation (13) and is plotted in Fig. 6:

$$
\begin{gathered}
\sigma(x)=\frac{7.12 \times 10^{7}}{4 \pi \epsilon_{o}}\left(\sum_{n=-M}^{M}\left(\frac{Q}{\sqrt{(x-n D)^{2}+a^{2}}}\right)-\sum_{n=-M}^{M}\left(\frac{\alpha Q}{\sqrt{(x-n D+d)^{2}+a^{2}}}\right)\right) ; \\
x \in\left(x_{1}, x_{6}\right), \text { Particel } / m^{2}
\end{gathered}
$$




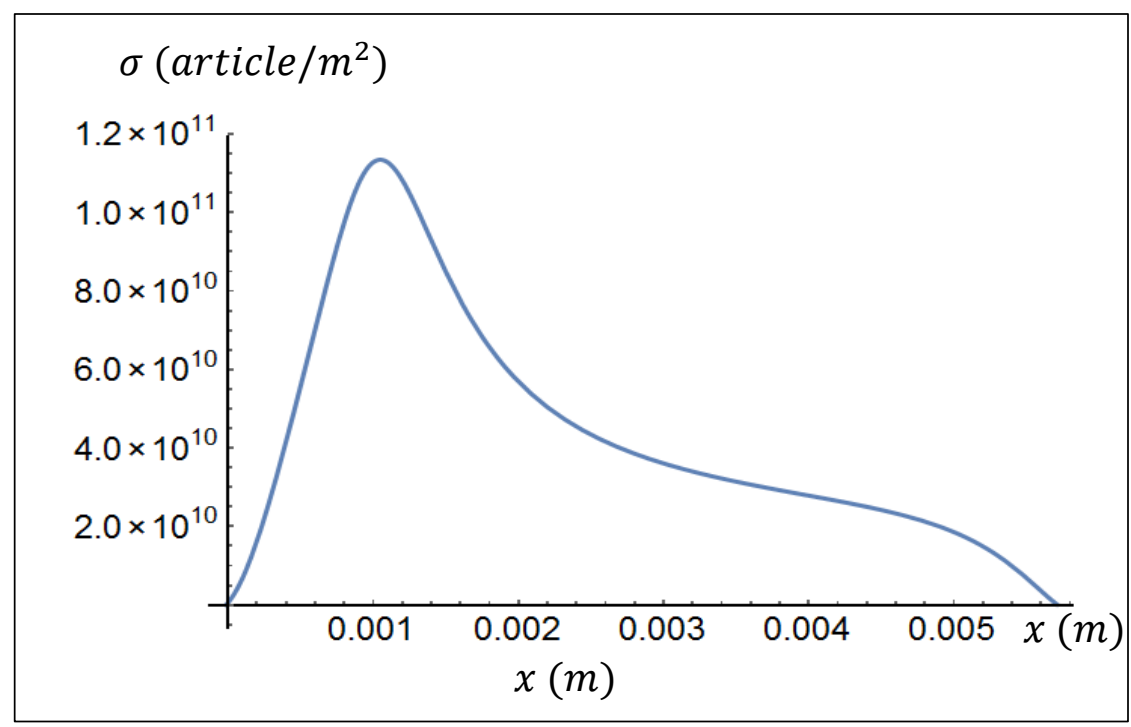

Fig.6: Positive Particles Distribution Function in the interval $x \in\left(x_{1}, x_{6}\right)$ in the $(y-z)$ plan.

\subsection{Positive Particles Transportation:}

The arrangement of SRP and PDF produce subsequent potential barriers (depletion regions) surrounding the positive electrodes as shown in Fig.2, the borders of these barriers are $\left(x_{1}, x_{2}, .\right.$. , $x_{6}$ ) depending on the equipotential value as illustrated in Fig. 1. The potential peak $\left(V_{B}\right)$ at these barriers and the borders of the depletion region will depend on:

1. The location of the SRP electrodes; $(d / D)$ ratio;

2. The ratio of charges mounted in positive electrodes to those in negative electrodes $(\alpha)$; and

3. The positive particles distribution (PDF).

The system setup confines the positive particles to transport in one degree of freedom in the $x$ axis, then depending on their kinetic energies and collision probability; the positive particles will have the probability to transport through the depletion region in wither $(+\hat{x})$ or $(-\hat{x})$ direction crossing the depletion region. To evaluate the transportation probability, imagine that the steady positive particles distributed as in the PDF given in Equation (13) start acquiring kinetic energies from the neutral particles by random collisions, the kinetic energy distribution of neutral particles will be subject to Maxwell-Boltzmann Distribution. The neutral particles collide randomly with the positive particles; hence, it is justified to assume that all positive particles will have averaged kinetic energy $\left(\bar{E}_{k}\right)$ from collisions with neutral particles.

The positive particles have mutual electric repulsive force, and then every positive particle that has a collision with neutral particle will make a field-field interaction with the other surrounding positive particles exerting the electric field of the collided positive particle. As a result of such collisions and electric field-field interactions, all positive particles will oscillate around their equipotential locations with displacements depending on:

1. Their location in the positive particle's distribution, where outer positive particles will have longer displacement oscillations; and 
- The kinetic energy acquired from both collisions with neutral particles and electric field-field interaction.

According to this configuration, there will be some $(y-z)$ plane of positive particles that the electric force in the $(+x)$ direction over this plane is equal to that in the $(-x)$ direction. Based on this "reflecting" plane, the positive particles located to the right of the plane will eventually transport part of their kinetic energy as waves toward $(+x)$ direction, and those located to the left of the plane will eventually transport part of their kinetic energy toward $(-x)$ direction.

To estimate the ( $x$-axis) location of this reflecting plane $\left(x_{r}\right)$, consider the PDF in Equation (13), then the electric force in the two sides of the $(y-z)$ reflecting plane:

$$
\int_{x_{1}}^{x_{r}} \frac{\sigma(x)}{\left(x-x_{r}\right)^{2}} d x=\int_{x_{r}}^{x_{6}} \frac{\sigma(x)}{\left(x-x_{r}\right)^{2}} d x
$$

Solving this equation gives $\left(x_{r} \cong 0.00107 \mathrm{~m}\right)$, which close to the location of the peak positive particle density as shown in Fig.6.

Defining the energy transportation ratio $\left(R_{E}\right)$ as the kinetic energy of positive particles transported in the $(+x)$ direction to the border $\left(x_{6}\right)$, to that kinetic energy of positive particle transported in the $(-x)$ direction to the border $\left(x_{1}\right)$. To evaluate $\left(R_{E}\right)$, the positive particles will be assumed transporting from their equipotential locations to the borders $\left(x_{1}, x_{6}\right)$ in the direction $(+x)$ or $(-x)$ based on their location relevant to $\left(x_{r}\right)$. During transportation, the positive particles will experience random collisions with neutral particles, statistically yielding an attenuation in the kinetic energy of the transporting positive particles, then:

$$
R_{E}=\frac{\int_{x_{1}}^{x_{r}} \sigma(x) e^{-\frac{\left(x-x_{1}\right)}{l}} d x}{\int_{x_{r}}^{x_{6}} \sigma(x) e^{-\frac{\left(x_{6}-x\right)}{l}} d x}
$$

Where $(l)$ is the mean free path of the neutral gas, from Equation (13) and the substituting $\left(x_{r} \cong\right.$ $0.00107 \mathrm{~m})$, the relation between $\left(R_{E}\right)$ and $(l)$ is shown in Fig.7, where $(\sigma(x))$ was expanded in polynomial function to the order (20) to avoid the integral of $\left(e^{x} / x\right)$ like function, the fitting was conducted using the least-squares method. The $\left(R_{E}\right)$ should have a value of unity for very small $(l)$ as well as for very large $(l)$, because of that in the case of a very small mean free path, most of positive particles will be stopped before reaching the borders, and for very large mean free path, most of positive particles will reach the borders, then $\left(R_{E}=1\right)$.

The best system performance will be for mean free path in the range of $10^{-4} \mathrm{~m}$ as predicted in the previous Ionized Gas Thermoelectric Generator (IG-TEG) study ${ }^{[23]}$, and according to Fig.7, the best $(l)$ for the operation of the device is in the range $\sim 3 \times 10^{-4} \mathrm{~m}$, in which agrees in the order with what imposed in IG-TEG study ${ }^{[23]}$. At this value of mean free path; the kinetic energy transfer 
in the $(+x)$ direction to the border $\left(x_{6}\right)$ is about (3) times to that in the $(-x)$ direction to the border $\left(x_{1}\right)$, i.e.:

$$
R_{E \max } \cong 3
$$

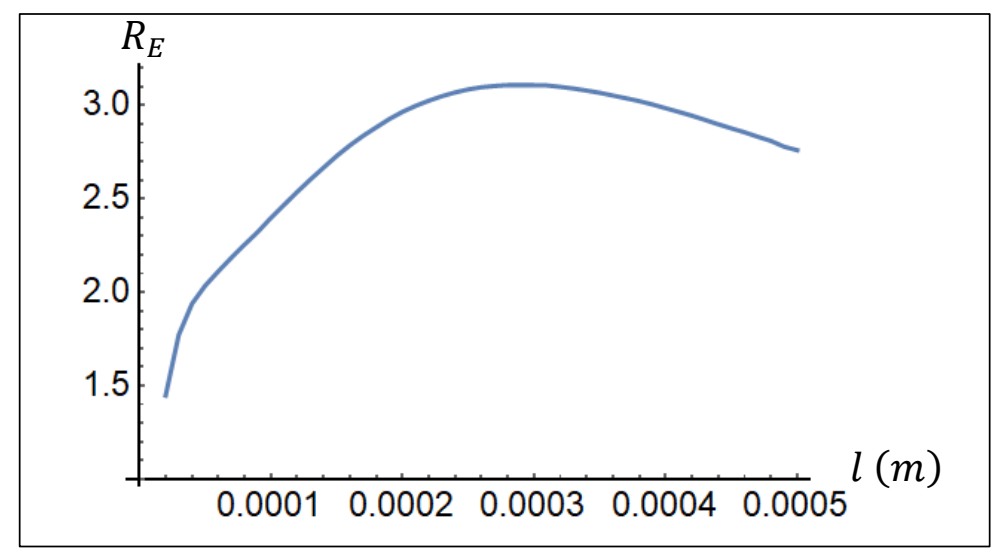

Fig.7: Relation between energy transportation ratio $\left(R_{E}\right)$ and mean free path $(l)$

The net kinetic energy transferred $\left(E_{n e t}\right)$ toward the border $\left(x_{1}\right)$, can be evaluated using Equation (16) below, mentioning that the initial positive particle kinetic energy will be assumed equal to the mean kinetic energy of the neutral particles, i.e. $\left(\bar{E}_{k}=\frac{3}{2} k_{B} T\right)$, hence the $(\mp x)$ components of the kinetic energy will be $\left(\frac{1}{2} k_{B} T\right)$, i.e. one degree of freedom. Then:

$$
E_{n e t}=\frac{1}{2} k_{B} T\left(\frac{N_{P}}{2}\right)\left(\frac{R_{E}-1}{R_{E}}\right) \int_{x_{1}}^{x_{r}} \sigma(x) \pi a^{2} e^{-\frac{\left(x-x_{1}\right)}{l}} d x
$$

Assuming $\left(\frac{1}{2} k_{B} T \sim 0.015 \mathrm{eV}\right)$, then:

$$
E_{\text {net }} \cong 7.69 \times 10^{6} \mathrm{eV} \cong 1.23 \times 10^{-12} \mathrm{Joule}
$$

As the energy of a net current of positive particles crossing the depletion region in the ( $-x$-axis) direction. In addition, the net power transfer will be:

$$
P_{\text {net }}=E_{\text {net }} \times \frac{\bar{v}}{\left(x_{r}-x_{1}\right)} \cong 1.43 \times 10^{13} \frac{\mathrm{eV}}{\mathrm{sec}} \cong 2.28 \times 10^{-6} \mathrm{Watt}
$$

Where $(\bar{v})$ is the average thermal speed of positive particles in the range $\left(x \in\left(x_{1}, x_{r}\right)\right.$ and to be considered $2 \times 10^{3} \mathrm{~m} / \mathrm{s}$ assuming the positive particles are ionized Hydrogen atoms $\left(\mathrm{H}^{+}\right)$. On the other hand, and taking into consideration the conservation of energy and conservation of linear momentum laws, a current of neural particles crossing the depletion region will be produced in accordance, equal in magnitude and opposite in direction, this neutral current will work as the energy carrier from the system to the outside in the energy harvesting stage. 


\subsection{Positive Particles Transportation in Depletion Region:}

The quantities of transported positive particles, the electric potential inside the depletion region, the energy transportation ratio, and the system total number of positive particles are correlated quantities. Hence, some averaging and approximations will be employed with justifications in the evaluation of the electric potential inside the depletion region, as well as during the evaluation of the number of transported particles.

Depending on the electric potential of the system (electrodes and positive particles), the transporting positive particles will pass through positive electrodes in certain trajectories forming a transportation tunnel, this tunnel is located inside the depletion region as illustrated in Fig.8(a). The neutral particles will be free to pass through the entire space of the depletion region. The length of the tunnel $(L)$ will be the same as the length of the depletion region $\left(L=x_{1}-x_{6}{ }_{6}\right)$ as shown in Fig.8.(b). The trajectories of positive particles are formed based on the minimal electric potential, forming the tunnel in a Cylindrical Anvil shape as shown in Fig.8.(a), with variable radius depend on the system electric potential and the energy transportation ratio and the number of transported positive particles.

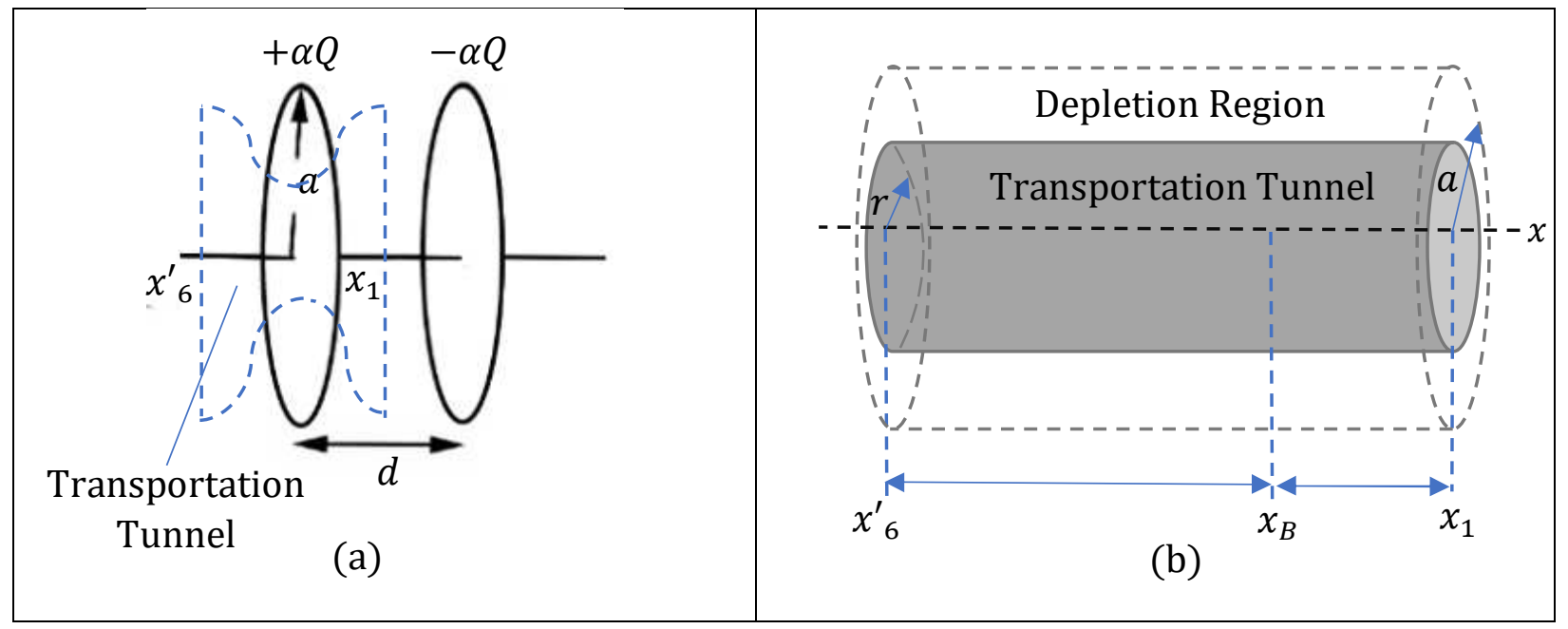

Fig.8: Transportation Tunnel

To facilitate the evaluation of the variable radius of the tunnel, assume that the tunnel is a cylinder with bases at $\left(x_{1}, x_{6}^{\prime}\right)$ as shown in Fig.8.(b), the radius of the tunnel is less than the radius of the electrode $(r<a)$, also assuming equal spacing between positive particles inside the tunnel to minimize and neglect the electric potential produced by the passing positive particles.

The cylindrical tunnel radius will depend mainly on the positive electrode potential, where the electric potential at the depletion region produced by the system electrodes varies gradually as moving away from $x$-axis toward $(y)$ and $(z)$ directions. Fig.9 shows the variation of electric potential at $(y-z)$ plane of the positive electrode, produced by few pairs of system electrodes. In Fig.9, the adjacent negative electrode approximated as a point charge located at a distance $(d)$ from the positive electrode, and the charge assumed as $(-\alpha Q)$ to represent the effect of positive particles near that negative electrode. 


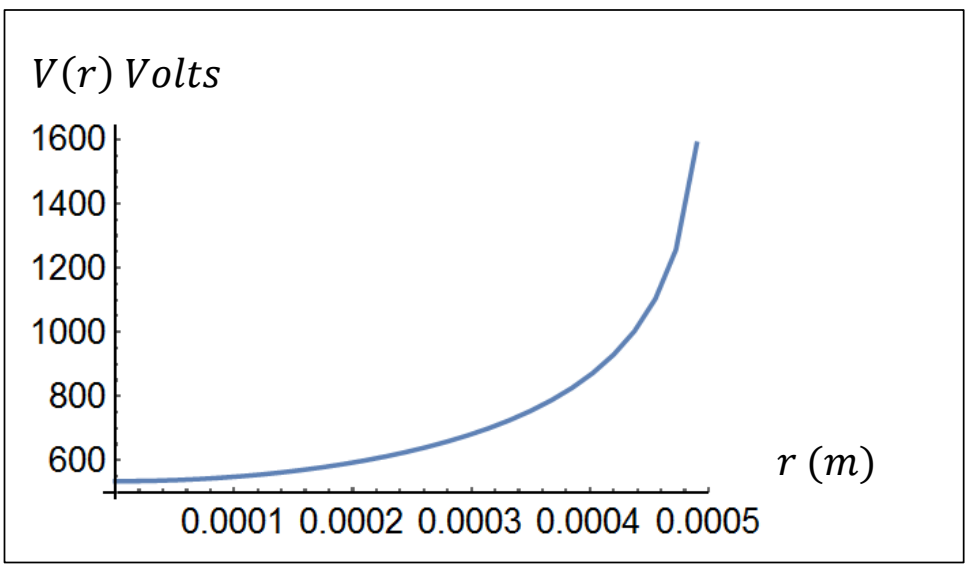

Fig.9: Electric potential at the positive electrode $(y-z)$ plane, where $\left(r=\sqrt{y^{2}+z^{2}}\right)$.

The positive particles will move in the trajectories with minimum electric potential, i.e. $(V \sim 353$ Volts) trajectory passing the center of the positive ring electrode.

From Fig.9, the minimum energy $\left(E_{T}\right)$ required for the positive particle to pass through the potential barrier (at $x=x_{B}$ ) is $\sim 530 \mathrm{eV}$ corresponding to a trajectory located exactly on the $x$ axis. According to the net power transfer $\left(P_{n e t}\right)$ evaluated in Equation (18), the maximum flow of the first pulse will be:

$$
I_{+, 0}=\frac{P_{n e t}}{E_{T}} \cong 2.67 \times 10^{10} \text { Particle } / \text { sec }-\hat{x}
$$

The maximum number of positive particles of this flow exist at the same time inside the depletion region will be:

$$
N_{+}=\frac{I_{+}}{\bar{v}_{i,+} /\left(x_{1}-x_{6}^{\prime}\right)}
$$

But the $\left(\bar{v}_{i,+}\right)$ varies with the electric potential inside the depletion region as:

$$
\bar{v}_{i,+}=\sqrt{\frac{2\left(E_{T}-q V(x)\right)}{m}}
$$

Where $V(x)$ is the electric potential at the $x$-axis inside the depletion region, noting that the minimum electric potential is $(V(x)=0)$ at the borders $\left(x_{1}, x^{\prime}{ }_{6}\right)$ of the depletion region, and the maximum electric potential at the $x$-axis is $\left(V\left(x_{b}\right)=E_{T} / q\right)$, hence the average speed of positive particles in the $\mathrm{x}$-axis $\left(\bar{v}_{i,+}\right)$ can be approximately assumed as:

$$
\bar{v}_{i,+}=\frac{1}{2} \sqrt{\frac{2 E_{T}}{m}} \cong 1.6 \times 10^{5} \mathrm{~m} / \mathrm{sec}
$$

This speed value is way far from the relativistic limits $\left(\sim 10^{-4} c\right)$, so that no need to consider relativistic analysis. Substituting the average speed in Equation (22) into Equation (20) gives: 


$$
N_{+}=43.4 \text { Particles }
$$

Existed inside the depletion region simultaneously. Considering the length of the depletion region, the electric potential produced by these positive particles will be in the range $1^{-3}$ Volts, so it will be neglected compared to the system electric potential $(>530 \mathrm{~V})$. As a result, the flow of positive particles will be passing through the depletion region in a single trajectory located at the $x$-axis.

\subsection{Neutral Particles Transportation:}

The neutral particles are existed in the depletion region by random Brownian motion, the Brownian density of neutral particles $\left(n_{B, \pm}\right)$ existed in the depletion region by Brownian motion can be evaluated based on the neutral particles mean free path $\left(l \sim 3 \times 10^{-4} \mathrm{~m}\right)$, and assuming that the neutral particles are Helium $(\mathrm{He})$ gas particles in the standard room temperature, with a diameter $(d=0.225 \mathrm{~nm})$, as:

$$
n_{B, \pm}=\frac{1}{l \pi d^{2}} \cong 2.1 \times 10^{22} \text { Particels } / m^{3}
$$

Additional neutral particles will exist in the depletion region, these additional neutral particles introduced to the depletion region by the net flow of neutral particles in the $(+\hat{x})$ direction explained in subsection 3.4. In accordance to the law of Conservation of Energy:

$$
\sum_{N_{ \pm}} \frac{1}{2} m_{ \pm} v_{ \pm}^{2}=\sum_{N_{+}} \frac{1}{2} m_{+} v_{+}^{2}
$$

Noting that $\left(m_{ \pm} \cong 4 m_{+}\right)$, then from Equation (25) and (19):

$$
I_{ \pm, 0}=\frac{I_{+, 0}}{2}=1.34 \times 10^{10} \text { Particle } / \mathrm{sec}(+\hat{x})
$$

The majority of collisions initiating the flow of neutral particles were occurred at the positive particles high-density region, a distance about $5 \mathrm{~mm}$ before the border compared to the mean free path $0.3 \mathrm{~mm}$. Hence, the energy gained by neutral particles from collisions with positive particles will be distributed equally among a large number $\left(\sim 2^{5 / 0.3} \sim 10^{5}\right)$ of positive particles, assuming that after each collision, two collided particles will share the collision energy. Then, the neutral particles flow can be assumed homogeneous and mono-energetic inside the depletion region. Then the density of neutral particles increment rate with time will be:

$$
\frac{d n_{ \pm}(t)}{d t}=\frac{1}{V} \frac{d N_{ \pm}}{d t}=\frac{I_{ \pm}}{V} \quad \text { Particles } / \text { Sec } . m^{3}
$$

Then:

$$
n_{ \pm}(t)=n_{B, \pm}+\frac{I_{ \pm} t}{V} \text { Partcile } / m^{3}
$$


Where $(V)$ is the volume of the transportation tunnel $\left(\pi a^{2}\left(x_{1}-x^{\prime}{ }_{6}\right) \cong 8.47 \times 10^{-10} \mathrm{~m}^{3}\right)$. Then the mean free path inside the transportation tunnel will be a function of time, as:

$$
l(t)=\frac{1}{\pi d^{2}\left(\frac{I_{ \pm} t}{V}+n_{B, \pm}\right)}
$$

The mean free path will increase with time, starting from the initial mean free path $\left(l \sim 3 \times 10^{-4} \mathrm{~m}\right)$ as shown in Fig.10. The increase in the mean free path inside the depletion region will decrease the positive particles current passing through the depletion region, hence decreasing the neutral current increments, and so on until reaching terminal currents $\left(I_{+}\right)$and $\left(I_{ \pm}\right)$.

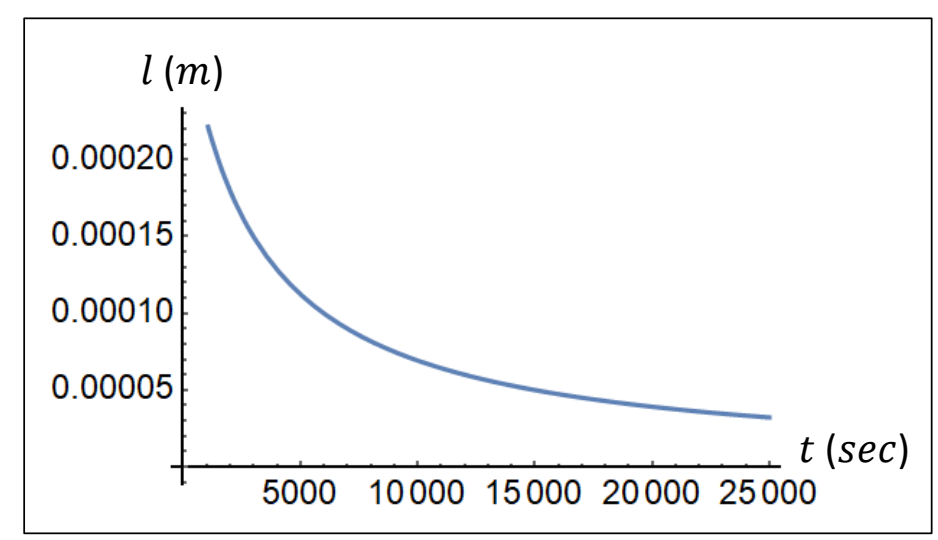

Fig.10: Mean free path time variation due to increasing of neutral particle flow.

\subsection{Positive Particles and Neutral Particles Terminal Currents:}

To evaluate the correlated terminal currents $\left(I_{+}\right)$and $\left(I_{ \pm}\right)$, a conservative approximation will be imposed to facilitate the evaluation, in which the positive particles current attenuation will be considered only before the voltage peak point $\left(x_{B}\right)$, where positive particles experience a collision after this point will not significantly affect the transportation of these particles because of the electric drifting force. Then the positive particles current attenuation will be:

$$
I_{+}=I_{+, 0} \operatorname{Exp}\left[\frac{-\left(x_{1}-x_{B}\right)}{l}\right]=I_{+, 0} \operatorname{Exp}\left[-\left(x_{1}-x_{B}\right) \pi d^{2}\left(\frac{I_{+} t}{2 V}+n_{B, \pm}\right)\right]
$$

This equation involves $(\operatorname{Exp}(x) / x)$ term, then the solution of this equation will be in the form of Lambert W Function (Omega Function $-W(z)$ ), as:

$$
I_{+}=\frac{2 V}{\pi d^{2} t x} W\left(\frac{\pi d^{2} I_{+, 0} t x}{2 V} \operatorname{Exp}\left[-\pi d^{2}\left(x_{1}-x_{B}\right) n_{B, \pm}\right]\right)
$$

For the current controlling parameters summarized in Table.2, where the distance $\left(x_{1}-x_{B}\right)$ was estimated from Fig.2; the resulting positive particles terminal current $\left(I_{+}\right)$can be found as shown in Fig. 11, where the foreseen timely current decrease with time shows slow current decay, as given for $10^{5}$ seconds in Fig. 11. 
Table.2: Current controlling parameters and their values

\begin{tabular}{|c|c|l|c|}
\hline \multicolumn{1}{|c|}{ Parameter } & Value (Unit) & Parameter & Value (Unit) \\
\hline $\begin{array}{l}\text { is the volume of the } \\
\text { transportation tunnel: } \\
(V)\end{array}$ & $8.47 \times 10^{-10}\left(\mathrm{~m}^{3}\right)$ & $\begin{array}{l}\text { Distance between border } \\
\left(x_{1}\right) \text { and voltage peak } \\
\text { location }\left(x_{B}\right) \text { in depletion } \\
\text { region: } \\
\left(x_{1}-x_{B}\right)\end{array}$ & $0.00013(\mathrm{~mm})$ \\
\hline $\begin{array}{l}\text { Helium }(\mathrm{He}) \text { gas } \\
\text { particles diameter: }\end{array}$ & $0.225(\mathrm{~nm})$ & $\begin{array}{l}\text { First pulse positive particle } \\
\text { current: } \quad\left(I_{+, 0}\right)\end{array}$ & $2.67 \times 10^{10}$ \\
$(\mathrm{~d})$ & Second & $\begin{array}{l}\text { Initial neutral particles } \\
\text { density in depletion region: } \\
\left(n_{B, \pm}\right)\end{array}$ & $2.1 \times 10^{22}$ \\
\hline Time: & \begin{tabular}{l} 
Particels $\left./ \mathrm{m}^{3}\right)$ \\
\hline
\end{tabular}
\end{tabular}

The slow decreasing in the positive current $\left(I_{+}\right)$can be explained based on the followings:

- The current resistance is related to the mean free path $(l)$, which depend on the variant number of neutral particles $\left(n_{ \pm}(t)\right)$ the depletion region as given in Equation (29);

- The value of $\left(n_{ \pm}(t)\right)$ depend on the variant neutral particles current $\left(I_{ \pm}\right)$as given in equation (28); and

- The neutral particles current $\left(I_{ \pm}\right)$is correlated to the value of the current $\left(I_{+}\right)$itself as given in Equation (26).

Then, once the positive particles current $\left(I_{+}\right)$decrease, the current resistance decrease as well. The resulting $\left(I_{+}\right)$from this correlated relation will be as shown in Fig. 11, where the positive particles terminal current will be decreasing with time in a slowing pattern as $(t \rightarrow \infty)$. This slowing attenuation can be explained by noting the slow decreasing of mean free path shown in Fig. 10, due to the slow increasing of neutral particles in the depletion region, where the relation between current attenuation and mean free path is expressed by Equation (30). The energy extraction can be executed early within the first 100 seconds by letting the neutral current escape from the system in tangential slits (while the positive particles current remains confined by electrodes potential to move inside the circular system trajectory), and then from Fig.11, the positive particles current can be assumed in the range $\sim 5 \times 10^{9}$ Particles $/ \mathrm{Sec}$.

Once the neutral current $\left(I_{ \pm}\right)$starts escaping from the system, the mean free path can be assumed constant as predicted from Equation (29). Hence, the maximum extracted power from the escaped neutral current $\left(P_{\max }\right)$ can be evaluated based on the linear relation between particles current $(I)$ and the power $(P)$ carried by this current (at constant pressure and volume, based on the Ideal Gas Law), as:

$$
\frac{P_{1}}{P_{2}}=\frac{I_{1}}{I_{2}}
$$


Hence, considering the result of that the positive particles current can be assumed in the range $\sim 5 \times 10^{9}$ Particles/Sec, and from Equations (18), (19) and (32):

$$
P_{\text {max }}=\frac{5 \times 10^{9}}{2.67 \times 10^{10}} \times 2.28 \times 10^{-6}=4.27 \times 10^{-5} \mathrm{Watt}
$$

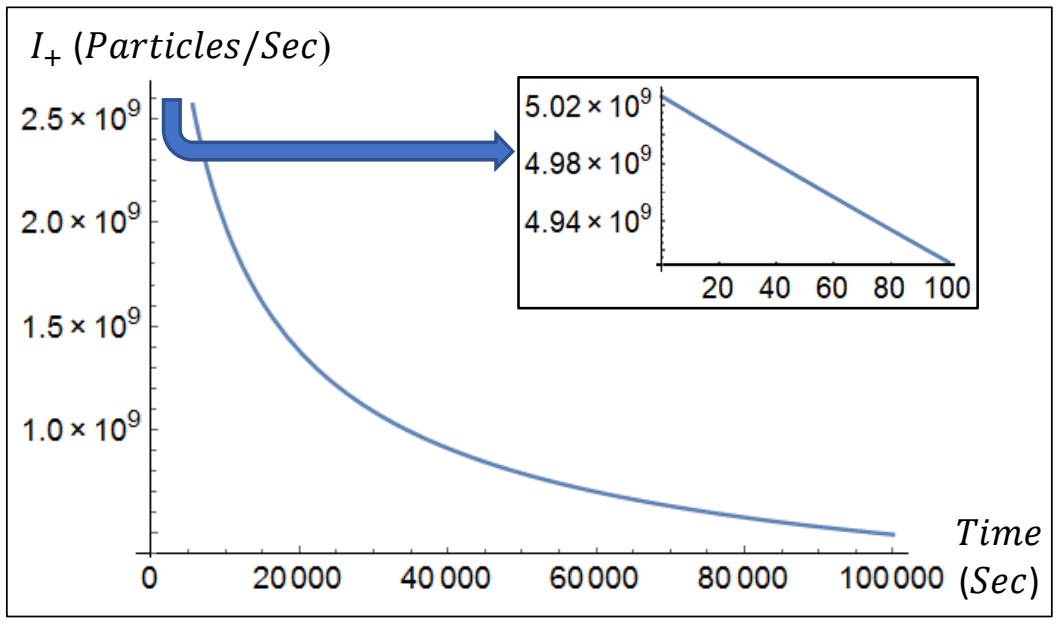

Fig. 11: Positive particles terminal current decreasing with time.

The result in Equation (33) can be used to estimate the power that can be extracted from a $1 \mathrm{~m}$ long system tube with a radius of $10 \mathrm{~cm}$, which considered a rational dimension that allows neutral particles flow to escape from the system tube, and based on the following conditions:

- Geometrical parameters of the electrodes given in Table.1; and

- Considering the same efficiency assumption (10\%) as explained in the previous study ${ }^{[23]}$.

The resulting power extraction will be in the order 10 Watts, which considered as a promising new green technology, and new effective cooling system that producing rather than consuming power.

\section{Conclusion.}

This study explains in computational manner the relation between the correlated parameters controlling the IG-TEG, including the geometrical parameters $(D, d, a)$, electrical parameters $(Q, \alpha Q, N q)$, and system pressure (neutral particles density and mean free path).

In this study, a new theoretical method was conducted to evaluate the power production of the IGTEG, and compared to the previous results ${ }^{[23]}$. The comparison shows agreement in the order of power extraction $\sim 10 \mathrm{Watt} / \mathrm{m}^{3}$. The new method was based on the evaluation of Positive Particles Distribution Function (PDF) in a given periodic interval of Static Ratchet Potentila (SRP). The PDF evaluaiton was based on $V=0$ equipotential locations of postive particles assumption, and extrapolating the volumetric distribution of positive particles from the linear distribution of particles, assuming uniformity in $y-z$ plane. The Gauss's flux theorem was applied on a linear sequent of $0.6 \times 10^{-10} C$ and $-1 \times 10^{-10} \mathrm{C}$ charged SRP electrodes. The evaluation of charge enclosed inside the Gaussian 
surface concluded that the total charge of free charged particles was $\sim 0.4 \times 10^{-10} \mathrm{C}$, carried by a total number of positive particles $\sim 2.5 \times 10^{8}$ per pair of SRP electrodes, yielding a correlated particle density in the order $\sim 10^{16} \mathrm{~m}^{-3}$. This result was verified thoroughly by the analytical method of Madelung Constant, assuming that the equipotential locations are laid in the basis of simple cubic lattice (SCL).

\section{References.}

[1] P. Reimann. Brownian motors: noisy transport far from equilibrium. Phys. Reports 361 (2002) 57-265.

[2] D. McDermott, C. Olson Reichhardt, C. Reichhardt. Collective ratchet effects and reversals for active matter particles on quasi-one-dimensional asymmetric substrates. Soft Matter, 12, 8606-8615 (2016).

[3] B. Roche, P. Roulleau, T. Jullien, Y. Jompol, I. Farrer, D. Ritchie, D. Glattli. Harvesting dissipated energy with a mesoscopic ratchet. Nature Communication 6:6738. DOI: 10.1038/ncomms7738 (2015).

[4] Z. Wang, Z. Jia, X. He. Net motion of a charged macromolecule in a ratchet-slit. Soft Matter, 9, 11107-11112 (2013).

[5] B. Lau, O. Kedem, J. Schwabacher, D. Kwasnieskiab, E. Weiss. An introduction to ratchets in chemistry and biology. Mater. Horiz. 4, 310318 (2017).

[6] G. Lavella, R. Morfino, M. Maharbiz. A synthetic Brownian ratchet architecture for creating tailorable chemomechanical nanomachines. Appl. Phys. Lett. 101(1) 0137031 (2012).

[7] I. Pavlyukevich, Y. Li, Y. Xu, A. Chechkin. Directed transport induced by spatially modulated Lévy flights. Journal of Physics A: Mathematical and Theoretical. 48 495004. (2015).

[8] K. Liao, S. Collins, V. Brus, O. Mikhnenko, Y. Hu, H. Phan, T. Nguyen. Electron ratchets: State of the field and future challenges. ACS Appl. Mater. Interfaces 11,1,1081-1087 (2019).

[9] J. Bader, R. Hammond, S. Henck, M. Deem, G. McDermott, J. Bustillo, J. Simpson, G. Mulhern, J. Rothberg. DNA transport by a micromachined Brownian ratchet device. Proceedings of the National Academy of Sciences of the United States of America. 96(23):13165-13169 (1999).

[10] A. Depperschmidt, N. Ketterer, P. Pfaffelhuber. A Brownian ratchet for protein translocation including dissociation of ratcheting sites. J Math Biol. 66(3):505-534 (2013).

[11] A. Zarrin, D. Sivak, A. Brown. Breaking time-reversal symmetry for ratchet models of molecular machines. Phys. Rev. E 99, 062127 (2019).

[12] A. Mitra, F. Ignatovich, L. Novotny. Nanofluidic preconcentration and detection of nanoparticles. J. Appl. Phys. 112.14304.10.1063/1.4731250. (2012).

[13] Q. Chen, J. Wu, C. Hu. Transport of interacting self-propelled Brownian particles in a common ratchet potential. Eur. Phys. J. B 88, 22 (2015). 
[14] C. Li, H. Chen, H. Fan, G. Xie, Z. Zheng. Transport of interacting self-propelled Brownian particles in a common ratchet potential. J. Physa. A.11.161 (2018).

[15] A. V. Plyukhin. Intrinsic ratchets: A Hamiltonian approach. Phys. Rev. E 98, 042130 (2018).

[16] M. Skaug, C. Schwemmer, S. Fringes, C. Rawlings, A. Knoll. Nanofluidic rocking Brownian motors. Science 359:1505-1508 (2018).

[17] N. Quintero, R. Alvarez-Nodarse, J. Cuesta. Ratchet effect on a relativistic particle driven by external forces. Journal of Physics A: Mathematical and Theoretical. 44 425205. (2011).

[18] S. Ethier, J. Lee, R. Soc. The flashing Brownian ratchet and Parrondo's paradox. Open Sci. 5:171685 (2018).

[19] V. Rozenbaum, I. Shapochkina, Y. Teranishi, L. Trakhtenberg. Symmetry of deterministic ratchets. Phys. Rev. E 100, 022115 (2019).

[20] S. Chowa, W. Huangb, Y. Lia, H. Zhoua. A free energy based mathematical study for molecular motors. Regul. Chaotic Dyn., 16:1-2,117-127 (2011).

[21] P. Malgaretti, I. Pagonabarraga, J. Miguel Rubi. Entropic transport in confined media: a challenge for computational studies in biological and soft-matter systems. J. Chem. Phys. 138, 194906 (2013).

[22] J. Rodenburg, S. Paliwal, M. Jager, P. Bolhuis, M. Dijkstra, R. Roij. Ratchet-induced variations in bulk states of an active ideal gas. J. Chem. Phys. 149, 174910 (2018).

[23] M. Bqoor. Ionized Gas Thermoelectric Generator. J.Tsep.100496 (2020).

[24] B. Crosignani, P. Di Porto. On the Validity of the Second Law of Thermodynamics. in the Mesoscopic Realm, EDP Sci. EPL (Europhys. Lett.) 53 (2001) 3.

[25] G. Wang, E. Sevick, E. Mittag, D. Searles, D. Evans. Experimental Demonstration of Violations of the Second Law of Thermodynamics for Small Systems and Short Time Scales. Phys. Rev. Lett. 89 (5) (2002).

[26] E. Purcell, D. Morin, Electricity and Magnetism, Cambridge University Press, UK, 3rd Revised edition, ISBN10 1107014026, ISBN13 9781107014022 (2013).

[27] D. Griffiths, Introduction to Electrodynamics, Cambridge University Press, UK, 4th Revised edition ISBN-10: 1108420419, ISBN-13: 978-1108420419 (2017).

[28] K. Hoffmann, K. Koch, T. Doerge, S. Micera. New technologies in manufacturing of different implantable microelectrodes as an interface to the peripheral nervous system. The First IEEE/RAS-EMBS International Conference on Biomedical Robotics and Biomechatronics, Pisa, 2006, pp. 414-419, 10.1109/BIOROB.2006.1639123 (2006).

[29] M. Niittymäki, K. Lahti, T. Suhonen, J. Metsäjoki. Dielectric Breakdown Strength of Thermally Sprayed Ceramic Coatings: Effects of Different Test Arrangements. Therm. Spray Tech. 24, 542-551 (2015).

[30] P. Misra, Physics of Condensed Matter, Academic Press, 1st Edition, ISBN-10: 9780123849540, ISBN-13: 978-0123849540, ASIN: 0123849543 (2011).

[31] R. Crandall, J. Delord. The potential within a crystal lattice. J. Phys. A: Math. Gen. 20 (1987) 2279-2292. 